\title{
How safe is our "place of safety"? Clinical guidance promoting safer medical care of patients detained under section 136 of the Mental Health Act
}

Josie Mouko, Aurielle Goddard, Victoria Nimmo-Smith

Avon and Wiltshire Mental Health Partnership NHS Trust, UK.

\begin{abstract}
A new four-bed unit was opened in Bristol, UK, in 2014, for people detained under section 136 of the Mental Health Act. Police bring individuals posing a risk to themselves or others to a Place of Safety (PoS) in order to receive a mental health assessment. Individuals may be held for up to 72 hours, but cannot receive treatment against their will, unless assessed as lacking the capacity to refuse treatment. Issues requiring medical input arose in more than a third of patients, yet there was little guidance for trainees around the PoS.
\end{abstract}

We conducted a survey which confirmed that robust clinical guidance was needed for junior doctors around medical assistance in this unique environment. We identified specific concerns around patient safety in relation to alcohol withdrawal, uncertainties around legislation and lack of clarity of who to call out of hours. Trainees felt they were working outside of their expertise.

We collaborated with a variety of professionals to produce clinical guidance in line with best evidence, and made this easily accessible. We also gained a consensus that more experienced core trainees (SHOs) in Psychiatry should be the first point of contact.

We then conducted a survey in June 2015, and found that doctors covering the PoS now felt there was sufficient guidance on most clinical scenarios, $100 \%$ consensus on who to contact and improved confidence in their ability to manage issues arising.

In August 2015 we held an informal training session for the new intake of trainees on the rota. A subsequent survey revealed similarly positive results.

Through this project, we were able to identify defects in a system, provide needed guidance to enable safer and more equitable care to a vulnerable group, and foster closer collaboration between junior doctors and managers in the design and use of services.

\section{Problem}

A four-bedded "Place of Safety" was opened in 2014 in Bristol, UK, by Avon and Wiltshire Mental Health Partnership NHS Trust, a large NHS provider of mental health care in the UK. This place of safety has over 1000 admissions a year.

It was identified anecdotally that junior psychiatrists on call were unclear around their roles, responsibilities, and the legalities of treatment for these individuals. Specific concerns were also raised around the management of alcohol withdrawal in the unit.

This project aims to identify problems trainees had encountered, clarify responsibilities of trainees, provide robust professional guidance and enable trainees to feel confident in providing care for these patients.

\section{Background}

If police officers are concerned that an individual in a public place is suffering from a mental disorder and requires care or control (in their interests or for the protection of others), they can be taken to a
"Place of Safety" (PoS). They are detained under section 136 of the Mental Health Act (MHA) for the purpose of having a psychiatric assessment and for appropriate aftercare arrangements to be made. Places of safety commonly in use are police cells and specially designed mental health "PoS/136 suites" (such as this one). There has been a commendable recent national drive to end detention of mentally disordered individuals in police cells under s136 due to this being seen as 'criminalising', distressing, and often de-humanising [1] and with little access to psychiatric or medical care [2].

Once individuals are in the PoS, they can be legally held there up to 72 hours, during which time physical health issues or disturbed behaviour may occur. The Royal College of Psychiatrists recommend that people detained under $\mathrm{s} 136$ of MHA should have a mental health assessment within two to three hours [3], to determine if they can be discharged or require further detention under section 2 or 3 of the MHA. This recommendation is supported in the MHA Code of Practice issued in 2015 [4].

Section 136 does not afford professionals any powers under the MHA to treat without consent, meaning that individuals on a section 136 are in the same position with respect to consent to treatment as 
patients not detained under the Act [4]. If a patient requires treatment and is lacking capacity they can be treated in their best interests under the Mental Capacity Act [5].

A four-bedded PoS was opened on a large NHS site (Southmead) which contains an A\&E department and general hospital as well as inpatient psychiatric facilities. This new unit replaced an individual site with one bed in Bristol. A variety of different approaches and policies, for example clinical management of disturbed behaviour, are used in different areas across the UK. The Royal College of Psychiatrists has issued guidance on the use and service provision of a place of safety $[2,3]$ but there is no available guidance nationally or locally on the appropriate management of clinical problems for detainees in the PoS.

Despite the Royal College guidance on time to MHA assessment, occurrences of prolonged stay in the PoS up to 72 hours can occur. During this time, patients may require medical review for alcohol detoxification, prescription of regular medications (such as insulin or anti-epileptics), agitated behaviour, regular medication, or acute physical health problems in a non-hospital setting. There may also be a need for training of staff on use of the mental capacity act for management of physical and psychiatric emergencies.

It was also identified that there was no existing consensus regarding which out-of-hours doctor was responsible for providing medical input to people detained on the PoS. Some appeared to be contacting the on call "junior" trainee (foundation doctors, GP trainees, and CT1s), however it appeared such junior staff lacked confidence and training to manage the complexities of the PoS.

Alongside ongoing efforts to reduce the length of stays for patients, it was felt by junior doctors and trust management that clear guidance on the roles and responsibilities of medical staff in managing physical health problems including management of alcohol withdrawal or disturbed behaviour in the PoS was necessary to ensure patient safety.

\section{Baseline measurement}

Two types of baseline measurements were taken. Firstly, we collected data over two months to determine the number of individuals on the unit who required medical attention and categorized them according to the indication for medical input. Secondly, we sent a survey to junior doctors in order to understand their experiences providing medical input to the PoS.

In two months there were 183 admissions. Of these a significant proportion (39\%) required some medical input during their stay. The reasons for requiring medical input were mainly for regular medication to be prescribed ( $n=31)$, for agitated behaviour $(n=25)$, pain relief $(n=17)$, or alcohol withdrawal $(n=8)$. More rarely there was an acute physical concern that developed while the detainee was on the unit $(n=5)$.

The survey identified the following problems for trainees who were providing out of hours medical input to people detained on the PoS:
1. Trainees were uncertain about management of common clinical scenarios such as agitation, alcohol dependence and withdrawal, prescription of regular medication.

2. All trainees agreed that additional guidance would be helpful.

3. Trainees were unclear about the legalities of prescribing in non-consenting patients.

4. $60 \%$ of surveyed trainees did not feel comfortable that they were working within their expertise, citing legal aspects and safety concerns as reasons for feeling uncomfortable.

5. There was a lack of clarity about whose role it is to provide medical cover; $20 \%$ believed junior $\mathrm{SHO}$ should be contacted, $40 \%$ believed senior $\mathrm{SHO}$ should be contacted, and $40 \%$ believed consultant on call should be contacted.

6. All trainees surveyed were clear how to access senior support.

See supplementary file: ds6148.docx - "Baseline measurements "

\section{Design}

We identified five specific outcomes to measure the efficacy of the interventions:

These were the extent to which junior doctors have:

a. Clarity on the first point of contact for medical input into Place of Safety.

b. Overall sufficient guidance.

c. Sufficient guidance around specific clinical scenarios (eg. agitation, alcohol dependence, regular medication).

d. Sufficient guidance around legal issues and the relevant use of the MHA and MCA.

e. Confidence that they were working within their expertise.

The baseline measures already showed that there was $100 \%$ clarity on where to get senior support. Because of this we did not make any intervention to target this.

The main intervention was to produce robust Professional Guidance for junior doctors for medical input to the PoS. This was done through reviewing current practice, consulting with clinical leads, junior doctors, and Approved Mental Health Professionals (AMHPs). Over several months, an iterative learning process was used with informal feedback through medical staffing meetings, junior doctor representation, and management meetings to create and modify the professional guidance, which was circulated in June 2015.

Part of the guidance was to develop a trust approved procedure for management of alcohol dependence and withdrawal in the PoS. This was done by jointly working with the trust drug and alcohol service and medicines management group. 
The guidance document was included inside the junior doctor on call handbook for the August 2015 intake of doctors, which is given out at the start of each year and was made available via the intranet. Prior to this the document was emailed to all junior doctors on the on call rota.

The second intervention was to determine an accepted procedure for contacting a doctor out of hours. Due to the clinical and legal complexities it was noted to be most appropriate for the senior $\mathrm{SHO}$ (psychiatry core trainee year two or three) to be the point of contact for medical concerns, with close liaison with consultants on call for areas of uncertainty or complex legal issues. This was then communicated through changing the on call rota names (clearly indicating which doctors covered the PoS), and making the junior doctor's handbook available on the intranet, via email to unit and on call staff and cascaded to the switchboard.

The above interventions were rolled out gradually midway through the year but were reinforced during the local induction in August 2015.

Following this we held training for the new intake of doctors in August.

\section{Strategy}

PDSA Cycle 1

The plan for the intervention was the issuing and disseminating of the place of safety professional guidance. After the initial baseline measurement (initial survey) we noted that there was confusion about the relevant use of the MHA vs MCA (mental capacity act) on the place of safety. For example, that a person with medical recommendation for a section 2 who is still in the Place of Safety could not be legally treated under the MHA. To fully evaluate whether clinical and legal knowledge improved we added an additional question to our survey for the next two rounds of measurements asking specifically about the practical use of the MHA and MCA.

We resurveyed the trainees to assess the efficacy of these interventions. Study results showed that while respondents had full clarity on who was responsible for providing medical cover there were still calls inappropriately going to the junior SHO. There was also some uncertainty about certain scenarios and the legal aspects. We found a difference between the confidence and competence of junior vs senior SHOs, which confirmed that this was the most appropriate procedure. Some respondents felt that further training and guidance would be useful. We also noted that there was no guidance on prescribing for methadone use and so we added this to the guidance.

PDSA cycle 2

At the start of the new training year August 2015 a new rota was sent to the switchboard. It had the new description of the $\mathrm{SHO}$ roles to include Place of Safety to ensure that calls go to the right person in the first instance. The guideline document and procedure was signposted during departmental induction.

We held a meeting and training session with trainees in order to get a better sense of the challenges trainees were encountering and to clarify the guidance around alcohol management. During discussion it transpired that there were some clinical scenarios for which prescriptive guidance could not be provided, such as managing selfharm.

We held training for and resurveyed only the senior SHOs on call during the August round of measurements. This was because the junior SHOs were no longer getting involved with the Place of Safety since the implementation of the new procedure.

We then held a deanery meeting to discuss the issues that were raised. It was identified that there was a perceived lack of supervision and oversight for trainees that was contributing to trainees' continuing feelings of unease when attending the Place of Safety.

See supplementary file: ds6600.docx - "Professional Guidance Place of Safety Document"

\section{Post-measurement}

Results following PDSA 1

There was $100 \%$ clarity on who the point of contact was for medical input to the PoS - senior SHO on call.

$80 \%$ of senior trainees but only $25 \%$ of junior trainees felt there was sufficient guidance around PoS, and that they were working within their expertise. $80-100 \%$ on senior trainees answered that they had adequate working understanding of various legal aspects (MHA vs $\mathrm{MCA}$ ) in PoS. In comparison the junior SHOs had less clarity around these legal issues.

$100 \%$ of senior SHOs felt there was sufficient guidance around managing most clinical scenarios (alcohol dependence and withdrawal, appropriateness of using anxiolytic and antipsychotics and prescription of regular medication). Junior SHO's were less clear, with many feeling there was insufficient guidance, which confirmed that the senior SHO was the most appropriate point of contact.

Results following PDSA 2

Following the face-to-face training in August 2015 we resurveyed the senior SHOs.

The 3rd survey showed broadly similar results to that of the previous senior SHO responses. There was however, more uncertainty around the use of the mental capacity act, managing self-harm or overdose and referral to $A \& E: 40 \%$ felt there was insufficient guidance about indications for referral to $A \& E$, managing self-harm or overdose on the place of safety and prescribing for individuals with and without capacity whilst in PoS). 
See supplementary file: ds6233.pptx - "Results following introduction of guidance"

\section{Lessons and limitations}

Our major learning points:

- How to overcome the difficulties and barriers in navigating a large organization, and a site with multi-agency stakeholders.

- A major positive outcome has been that this project fostered closer links between trainees and managers in the design and use of services.

Limitations:

- Our final survey was taken after the training session in August. As a result the trainees had only been working on the rota for one month whereas the survey in June was after SHOs had been working for almost a year. This could cause an underestimation of the usefulness of the training session, as the new starters would have been less confident than the respondents in June.

- There were some clinical areas that were reported as not being sufficiently covered in the guideline document: these were the management of self-harm or overdose and referrals to $A \& E$. These areas required doctors to exercise their clinical judgment in accordance with each situation and we felt that being too deterministic or prescriptive with the guideline could be unsafe. We discuss future plans to address this in the conclusion.

- The response rates have been relatively low to this survey (around $50 \%$ ). In addition, some responses were n/a if they had not had adequate experience to be able to answer. Therefore, the responses can only be taken as indicative and may be biased. For example, those who have had a particularly negative experience may be more likely to respond. Each group that we analysed had 5-10 respondents answering the survey so the power will be low.

- This project only focused on training two cohorts of doctors. However, to ensure sustainability we have made the guidance readily available to trainees on the trust intranet and to nursing staff working on the unit. We are developing a video presentation to be shown at all future junior doctor induction programmes, including information about where to find the additional guidance.

- The variables influencing patient safety are multifactorial in this unit. As a result directly measuring patient outcome and adversity, such as through incident reporting, was not feasible or appropriate. Additionally the frequency of adverse incidents were so low that to study this would require a very long study period. The ultimate aim of this project was to improve safety and equity for patients on the place of safety. Therefore, the use of the survey as a measure relies on the assumption that there is a correlation between junior doctor's perception of and actual patient safety. While we hope this is the case, it has not been proven.
- The production of this guidance did not incur any significant costs, due to the guidance being developed by trainees in their own time. Potentially, our project may have resulted in some efficiency savings for junior doctors, through providing clear and accessible guidance and training; however, we cannot identify a reliable way to measure such savings, due to the variety of confounding factors such as doctor experience and patient presentations.

- Finally, as the service is new it has been rapidly evolving in parallel with this project. Therefore potential improvements to trainee experience and patient safety are likely to also be influenced by the improvements in the service as a whole. For example, the unit signed up to a service enabling them to access GP records, this is likely to have had an impact on the safety of prescribing independent of the availability of the guideline document that we produced.

\section{Conclusion}

The aims of our interventions were to clarify responsibilities of trainees, provide robust professional guidance for trainees and enable trainees to feel confident in providing care for these patients. Over all we feel that we have achieved our aims and have identified further areas for improvement, which we have begun to address. The introduction of professional guidelines for the place of safety, in particular evidence based guidelines for alcohol withdrawal have been widely welcomed.

As far as we are aware, there have not been any similar studies looking at the provision of medical care to patients in a PoS. Guidance in other units and from the Royal College of Psychiatrists focuses on the responsibilities of medical staff around the psychiatric assessment of patients.

It is unclear whether other units around the country require such detailed guidance, as this only becomes necessary where patient stays are prolonged, as occurred for a proportion of patients on our unit. Other units have policies stating that if urgent medical assessment or treatment is needed, the detainee should be transferred to the A\&E department, which may be sufficient if mental health assessments by Section 12 doctors and AMHPs are occurring within the first few hours of detention.

As more PoS are opened - as the Royal College of Psychiatrists recommends - guidelines and procedures such as these are likely to prove essential for the safe running of these units.

This project provided invaluable insight for trainees to the complexities of management and leadership, and how to produce changes in a large organisation. Our project evolved and led to the development of regular meetings with trainees, deanery, medical education, and multi-agency working groups. These provided a forum to raise concerns and enabled trainees to be better informed about upcoming changes.

Future plans include a regular supervision group, enabling trainees to have a forum to discuss, share, and learn from individual experiences which do not lend themselves to directive guidance. It 
has also been agreed that senior psychiatrists with expertise in the PoS (who cover this unit during daytime hours) can be contacted to discuss specific issues arising.

We hope that this project has enabled trainees to provide more consistent, safer, and equitable care to a very vulnerable group of individuals, and led to an increase in confidence around providing this care. We hope it will be a useful starting point for other organizations facing similar challenges.

\section{References}

1 Department of Health. Review of the Operation of Sections 135 and 136 of the Mental Health Act 1983. Report Summary and Recommendations; 2014.

2 Royal College of Psychiatrists. Standards on the Use of Section 136 of the Mental Health Act 1983 (England and Wales): College Report CR159; 2011.

3 Royal College of Psychiatrists. Guidance for commissioners: Service provision for Section 136 of the Mental Health Act 1983: Position Statement PS2/2013; 2013.

4 Department of Health. Mental Health Act 1983: Code of Practice. $16.72 ; 2015$.

5 Department of Health. Mental Capacity Act 2005: Code of practice. $5.1 ; 2014$.

\section{Declaration of interests}

All authors have nothing to declare.

\section{Acknowledgements}

We would like to thank the following people for providing their expertise in developing the guideline document: Dr Rosie Herbert, Dr Ben Watson, Dr Amy Green, Dr Jacek Kolsut, and Rachael Searle-Barnes.

We would like to thank the following people for their encouragement and support in this project: our mentor, Dr Andrew Brindley, Dr Linda Heaney and Dr Rob MacPherson, Dr Dan Hodgson, Mark Bunker, and Dr Harvey Rees for their support and encouragement throughout this project, and in enabling enhanced links between trainees and trust management.

\section{Ethical approval}

Our project was an Improvement Study, with the main intervention being providing professional guidance which was in line with good practice. Our data collection required only surveying staff, with no direct intervention with patients. According to local policy at the time of conducting our project, it was not necessary to obtain ethical approval. 